\title{
Le syndrome douloureux régional complexe (SDRC) en 2012
}

\section{Complex regional pain syndrome (CRPS) in 2012}

\author{
B. Barrois \\ (C) Springer-Verlag France 2012
}

Prendre en charge des patients porteurs de SDRC reste un défi quasi quotidien pour la plupart des médecins MPR et de leurs équipes paramédicales. Dès le diagnostic posé, il faut rapidement mettre en œuvre un traitement. Néanmoins, peu de traitements sont validés par l'Evidence Based Medecine. Il est donc indispensable de faire régulièrement un point d'actualisation des connaissances pour cette pathologie et c'est l'objet de cette Lettre.

Les SDRC de type 1 arrivent aux consultations ou dans les services de MPR après un parcours plus ou moins long et plus ou moins chaotique selon l'étiologie qui a précédé la survenue de la pathologie. Les chirurgiens orthopédistes et les rhumatologues les repèrent aisément, de même que les médecins traitants quand il s'agit d'une étiologie fréquente de SDRC. Ils font alors en général appel à nos équipes quand le traitement de première intention n'est pas efficace.

En revanche, les cas plus rares qui, par exemple, compliquent la grossesse ou qui surviennent en pédiatrie peuvent être vus tardivement après le début des symptômes.

Les SDRC de type 2 sont souvent vus précocement mais leur traitement n'en est pas pour autant plus aisé.

Ces patients attendent de nos soins des réponses rapides et efficaces toujours complexes à apporter.

Depuis le début du $\mathrm{xx}^{\mathrm{e}}$ siècle et les recherches de Leriche et de Sudeck, des travaux émergent très régulièrement pour apporter de nouveaux regards sur la physiopathologie, sur les possibilités thérapeutiques médicamenteuses, sur les thé- rapies physiques et sur les possibilités d'actions sur le versant psychocomportemental.

Cette revue a pour objet de faire un état des lieux en 2012. Néanmoins, tous les axes ne seront pas balayés de façon exhaustive.

Une large revue des connaissances sert de base aux approfondissements plus ciblés (C. Palazzo).

L'analyse de l'actualité la plus récente sur la physiopathologie est envisagée et des critères diagnostics objectifs validés sont proposés (A. Berquin).

Cela précède une revue sur les possibilités thérapeutiques médicamenteuses (P. Calmels).

Quelques équipes font des études ciblées intéressantes aboutissant à des choix spécifiques émergents (M. Sorel).

Bien entendu, une très large place est faite aux programmes de rééducation orientés vers les techniques innovantes. Ils sont fondés sur les recherches les plus récentes, axées sur la négligence ou l'imagerie motrice (Y. Rossetti, B. Rioult).

L'analyse de nouvelles études sur la psychologie et la psychopathologie apporte un éclairage complémentaire (M.-C. Defontaine Catteau)

Les travaux spécifiques des SRDC des enfants et des adolescents ne sont pas traités dans cette revue.

Nous espérons que vous trouverez dans ce numéro de $L a$ Lettre de MPR quelques pistes pour répondre aux problèmes que vous posent certains patients et nous vous souhaitons une bonne lecture.
B. Barrois $(\square)$

Chef de service, service de MPR, centre hospitalier de Gonesse, BP 30071, F-95503 Gonesse

e-mail : brigitte.barrois@ch-gonesse.fr 